\title{
Advanced use of a split Hopkinson bar setup for the extended characterization of multiphase steel sheets
}

\author{
Joost Van Slycken ${ }^{1, a}$, Patricia Verleysen ${ }^{1, b}$ and Joris Degrieck ${ }^{1, c}$ \\ ${ }^{1}$ Department of materials science and engineering, Ghent University, St.-Pietersnieuwstraat 41 , \\ Ghent, Belgium \\ ajoost.VanSlycken@UGent.be, ’Patricia.Verleysen@UGent.be, ㄱoris.Degrieck@UGent.be
}

Keywords: High strain rate behavior, split Hopkinson bar, TRIP steel, tensile, compression, bending experiments

\begin{abstract}
In this paper some highlights are presented of an integrated numerical and experimental approach to obtain an in-depth understanding of the high strain rate behavior of materials. This is illustrated by an investigation of the multiphase TRansformation Induced Plasticity (TRIP) steel. 'Classic' high strain rate tensile experiments using a split Hopkinson tensile bar setup are complemented with strain rate jump tests, tensile tests at elevated temperatures and interrupted experiments. High strain rate compression and three-point bending experiments are performed on the steel sheets as well. The results reveal the excellent energy-absorption properties in dynamic circumstances of TRIP steels. Advanced experimental setups using the Hopkinson principle provide indeed tools for validation of the material and structural properties of TRIP steels.
\end{abstract}

\section{Introduction}

In order to optimize the crashworthiness analysis in the automotive industry, high strain rate testing of steels is becoming more important. It is well known that steels display a positive strain rate performance, i.e. at the higher rates of strain which are typically associated with crash events, steels have higher strengths and consequently a higher energy absorption potential for a crash event. This experience is already used in the design of the lightweight ULSAB-AVC car body structure [1].

The determination of the material properties over a large range of strain rates is thus a key step towards the accurate understanding of vehicle crash tests and of several high speed forming processes. This can for example be done by making advanced use of a split Hopkinson setup [2] and by combining a wide array of experiments with numerical modeling to study the high strain rate behavior of the material.

The approach will be illustrated by an investigation of the multiphase TRansformation Induced Plasticity (TRIP) steel [3]. This steel grade is a key member of the new generation steel grades and exhibits a combination of high strength and excellent (de)formability, resulting in a high energy absorption potential. This results from the complex synergy between the different phases in the steel grade and from the transformation of meta-stable retained austenite into martensite during deformation [4].

An extensive experimental program is set up to investigate the strain rate dependent mechanical properties of various TRIP steel grades. Static and dynamic experiments, with strain rates varying from 500 to $2000 \mathrm{~s}^{-1}$, are performed on a wide array of TRIP steel grades with varying alloying elements (Al, Si, P). Several microstructural observation techniques are used to reveal the mechanisms governing the observed high strain rate behavior. Special attention is paid to the temperature dependent behavior of TRIP steels and of the austenite to martensite transformation. 


\section{Experimental setup}

In order to obtain an in-depth understanding of the behavior of TRIP sheet steels an array of specially designed high strain rate experimental setups based on the Hopkinson principle is used. The overall material characterization is performed on the high strain rate Split Hopkinson Tensile Bar (SHTB) setup available at Ghent University (Fig. 1).

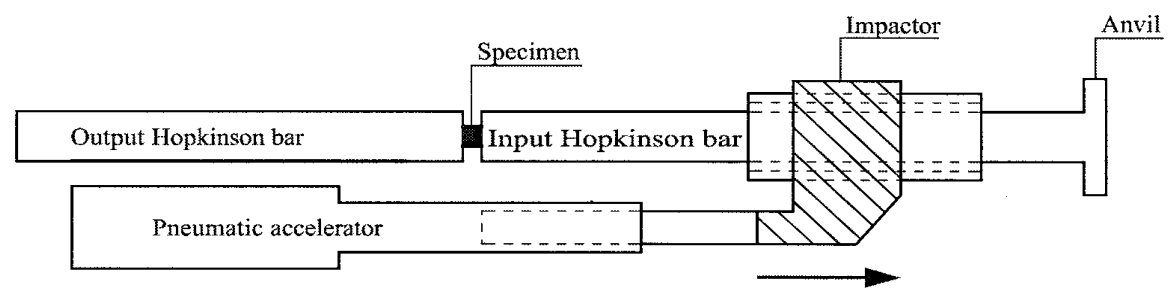

Figure 1: Schematic representation of the Split Hopkinson Tensile Bar (SHTB) setup

The setup mainly consists of two bars: an input and an output bar between which a specimen of the test material is attached. A tensile wave is produced by an impactor that is accelerated towards the anvil of the input bar. After the impact, this incident wave travels along the input bar towards the specimen where it interacts with the sample and is partly reflected back into the input bar. The other part, the transmitted wave, travels along the output bar. The strain histories of the different waves (incident, reflected and transmitted wave) are recorded by means of strain gauges mounted on both bars. By adjusting the impact speed of the impactor, the strain rate can be varied. According to the one-dimensional wave theory and the assumption of a uni-axial and homogeneous stress and strain in the specimen, the stress, strain and strain rate in the specimen can be calculated [5]. An extensive discussion and several critical remarks on high strain rate testing can be found in [6].

Based on this existing split Hopkinson tensile bar technique, more advanced tensile testing methods are developed by altering some testing features. During strain rate jump experiments, a sudden change in the strain rate is induced by using a specially shaped impactor. These experiments are particularly important in the determination of constitutive relations of the material behavior because the microstructure is supposed to have no significant evolution in the course of the rapid change of strain rate. Tensile tests at elevated temperatures $\left(20-80^{\circ} \mathrm{C}\right)$ are also performed. This additional temperature rise affects the material behavior and especially the austenite to martensite transformation. With interrupted SHTB experiments the specimen is not loaded until fracture but until a certain deformation level. In this way the microstructure and especially the martensite transformation at different stages of the plastic deformation can be observed. With a succession of short interrupted adiabatic experiments, where the specimen can be cooled down between two succeeding experiments, it is also possible to obtain an approximation of an isothermal high strain rate experiment [7]. Since the loading time of the specimen is proportional to the length of the impactor, a shorter impactor is necessary. Moreover, in these experiments the residual stress waves in the Hopkinson bars need to be absorbed to avoid additional loading of the specimen.

The high strain rate tensile testing program on sheet steels is completed with compression and bending experiments based on the Hopkinson principle. Although compression tests are widely used for dynamic characterization, this technique is not straightforward for in-plane testing of steel sheets because the material tends to buckle (Fig. 2a). Special cylindrical specimens are developed from a sandwich made out of glued thin steel sheets.

A behavior close to a real deformation mode of a structure can be evaluated by introducing a more dimensional stress state in the test specimen using a three-point bending setup (Fig. 2b). This setup is basically the same as the compression setup, yet two Hopkinson output bars are used. The moving input bar forces a rectangular specimen to deform out-of-plane. The use and implementation of these advanced dynamic testing techniques is however not straightforward. A detailed description and the experimental implementation of these setups can be found in [6]. 


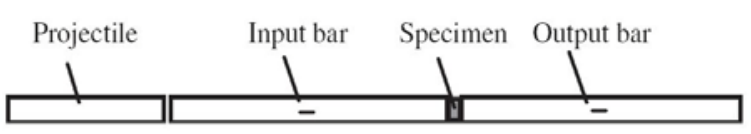

(a)

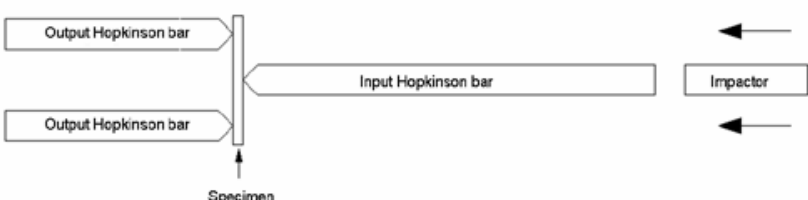

(b)

Figure 2: Schematic representation of the Split Hopkinson Pressure Bar (SHPB) setup (a) and the Split Hopkinson three-point bending Bar (SH3pbB) setup (b)

The composition of the investigated TRIP steels can be divided into four groups, based on the main alloying element: Si-based TRIP steel (CMnSi-TRIP), Al-based TRIP steel (CMnAl-TRIP), CMnSiAl-TRIP steel (based on full or partial replacement of Si by Al) and CMnSiAlP-TRIP steel with additions of P. Specific care was taken to keep the same carbon content for each steel grade. For the Al-based TRIP steel, two grades are considered with a different carbon content. CMnAlTRIP-LC represents the Al-based TRIP steel with the Lowest Carbon content. Two steels are industrially produced (TKS and ArcelorMittal), the other grades are laboratory steel grades [6].

\section{Classic dynamic tensile experiments}

The results of the static and high strain rate experiments show clearly that the excellent mechanical properties are not only preserved at higher strain rates, but they are still improved. In dynamic conditions the strain rate has limited influence on the material properties. Yet an important increase is noticed when comparing static to dynamic conditions (Fig. 3a). The differences in strength, elongation and energy absorption levels observed between the investigated materials can be attributed to their chemical composition (Fig. 3b). Silicon contributes to a significant solid solution strengthening and thus high strength levels. TRIP steels mainly alloyed with aluminum on the other hand exhibit lower strength values but higher elongation levels [8].

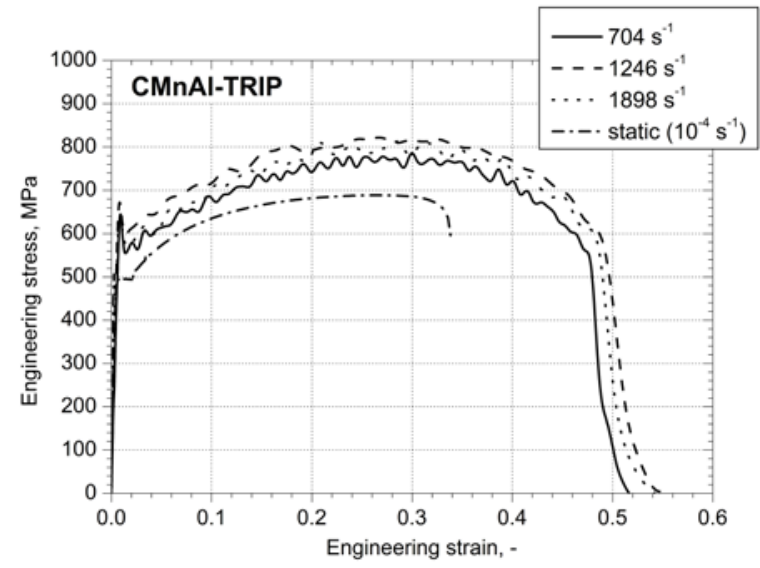

(a)

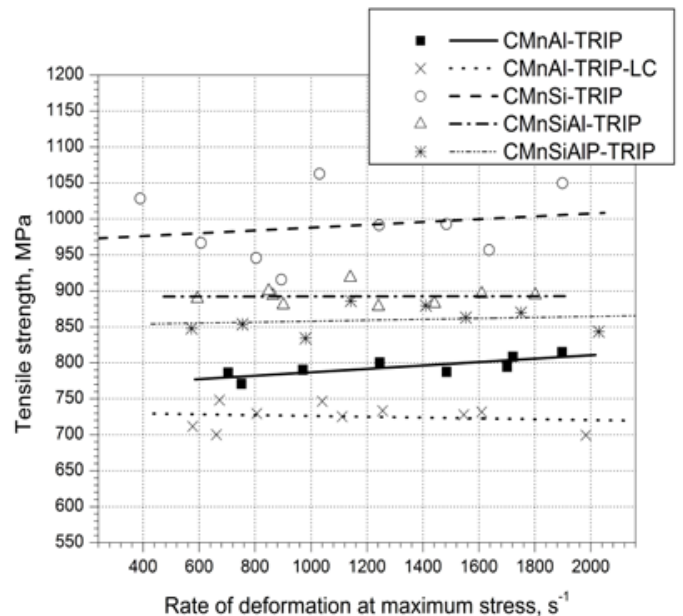

(b)

Figure 3: Representative engineering stress-strain curves for the Al-based TRIP steel grade after static and dynamic deformation. The indicated strain rate is the value reached at maximum stress

\section{Advanced dynamic tensile experiments}

For the materials investigated here, the strain rate jump has limited influence on the observed behavior and the effects are ranged within the experimental scatter. No significant influence on the material models is thus expected [6]. Higher testing temperatures cause an important decrease of the stress levels and energy dissipation in the industrial Al-based TRIP steel (Fig. 4). 


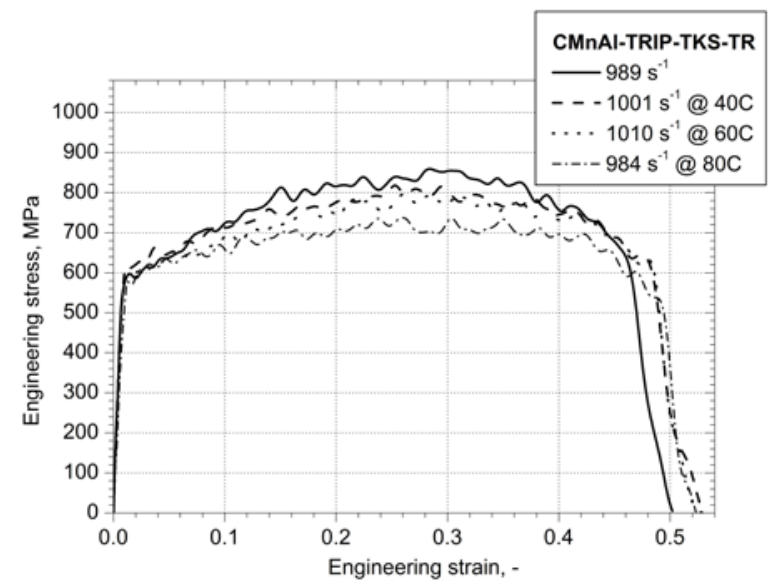

Figure 4: Influence of the testing temperature on representative high strain rate stress-strain curves for an industrial Al-based TRIP steel

The experiments learn that at $10 \%$ of deformation, $25 \%$ of the retained austenite has already transformed to martensite, whereas after full deformation $50 \%$ of retained austenite is transformed. These observations complement the results of the Light Optical Microscopy (LOM) evaluation of the microstructure evolution of the statically and dynamically deformed Al-based TRIP steel (Fig. 5). The elongation of the ferritic constituents increases close to the fracture, moreover this elongation is found to be strain rate dependent: higher strain rates result in more deformed grains. The austenite islands adopt a particular behavior as well: for static loading the austenite is slightly deformed and almost fully transformed. Whereas for dynamic loading on the one hand the austenite is highly deformed and transformed close to the fracture and it is slightly deformed and partially transformed away from the fracture on the other hand. The strain induced martensitic transformation occurs gradually in the material, letting time to the austenite to deform and bring more elongation to the TRIP steel. Adiabatic heating during high strain rate deformation tends to slow down the strain induced martensitic deformation [9].

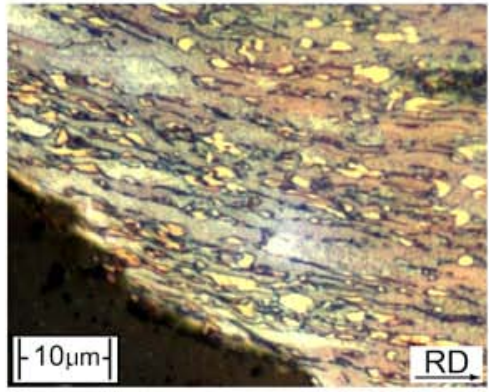

$\mathrm{x}=4.1 \mathrm{~mm}$

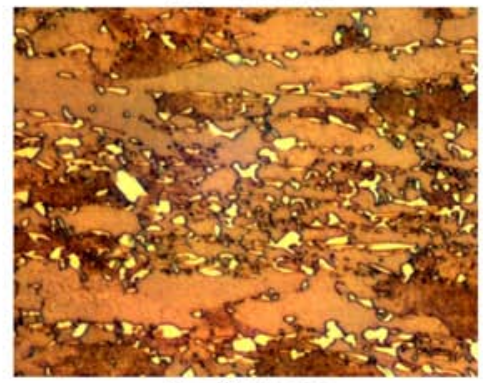

$\mathrm{x}=2.5 \mathrm{~mm}$

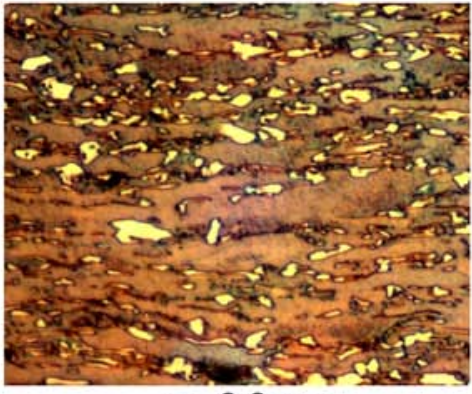

$\mathrm{x}=3.9 \mathrm{~mm}$

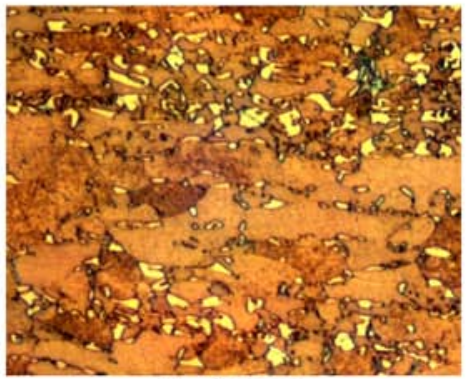

$\mathrm{x}=1.5 \mathrm{~mm}$

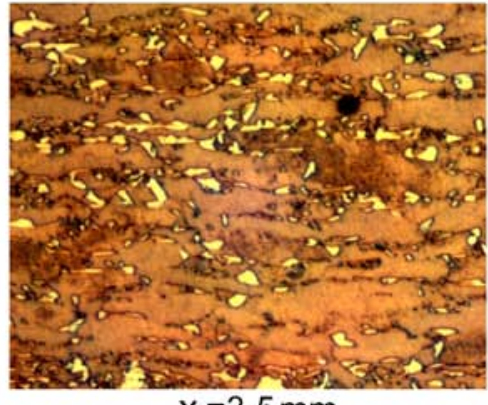

$\mathrm{x}=3.5 \mathrm{~mm}$

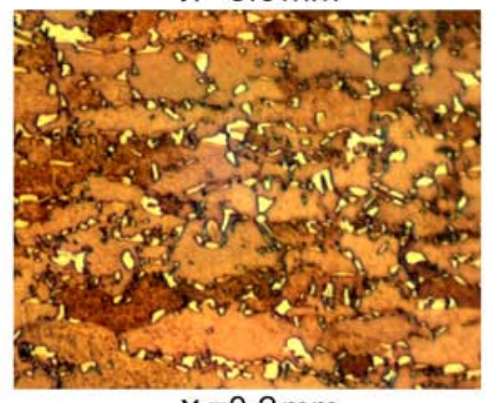

$\mathrm{X}=0.2 \mathrm{~mm}$

Figure 5: Al-based TRIP steel microstructure along the gage length of the specimen after a high strain rate test at a strain rate of $1323 \mathrm{~s}^{-1}$ (magnification: $\mathrm{x} 625$ )

\section{Dynamic compression testing}


The general conclusion of the FE calculation of the Split Hopkinson Pressure Bar (SHPB) experiment is that firstly, friction should be avoided as much as possible, whereas (accidental) indentation of the Hopkinson bar ends plays a minor role. Secondly, short specimens (height=diameter $<5 \mathrm{~mm}$ ) are preferred for testing because of a better uni-axiality distribution (Fig. 6a) [6]. From the experiments and the simulation it can be concluded that forces on the glue layer are very important and that this glue layer tends to fail rapidly (Fig. 6b). Lubrication and specimen length play a very important role in the observed behavior of the specimens. Based on a combined experimental/numerical approach a clear view of the dynamic compression behavior of TRIP steel can be given.

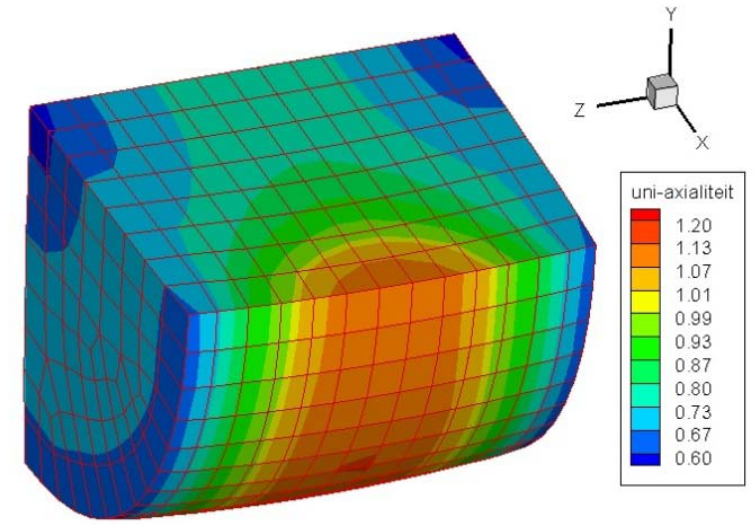

(a)
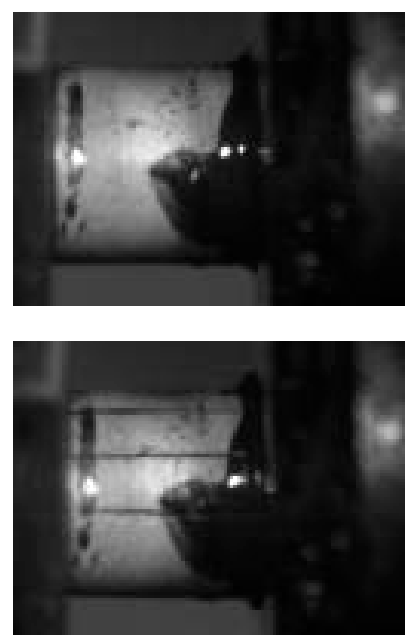

(b)

Figure 6: Distribution of the uni-axiality of the stress in a compression specimen (a) and high speed video recording of a compression experiment: the glue layer in the middle of the specimen is the first to fail (b)

\section{Dynamic bending testing}

As can be seen in Fig. 7a it is very difficult and complex to interpret the strain gauge signals of the Split Hopkinson three-point bending Bar (SH3pbB) experiment. Therefore it is necessary to investigate the specimen behavior using FE calculations. Fig. $7 \mathrm{~b}$ gives the force-relative displacement curves for different simulated materials. Besides the Johnson-Cook material model [10] of a Si-based TRIP steel (ArcelorMittal), used as a reference, five other material characteristics based on the Hollomon equation [11] are simulated in order to determine the influence of the material on the obtained results. It can be clearly seen that this force-displacement curve is indeed dependent on the material properties of the simulated model.

Common alignment imperfections are also simulated to better understand the measured signals during a real experiment. Small imperfections can significantly influence the signals, but the global shape of the curve is preserved as long as the imperfection is relatively small. Great care during aligning is thus required to repeatedly gather qualitative signals. Plastic deformation during the loading is concentrated in the centre of the specimen, the specimen acts in this way as a plastic hinge. It can be concluded that the SH3pbB setup is a possible validation tool for material and structural behavior of the investigated materials [6]. 


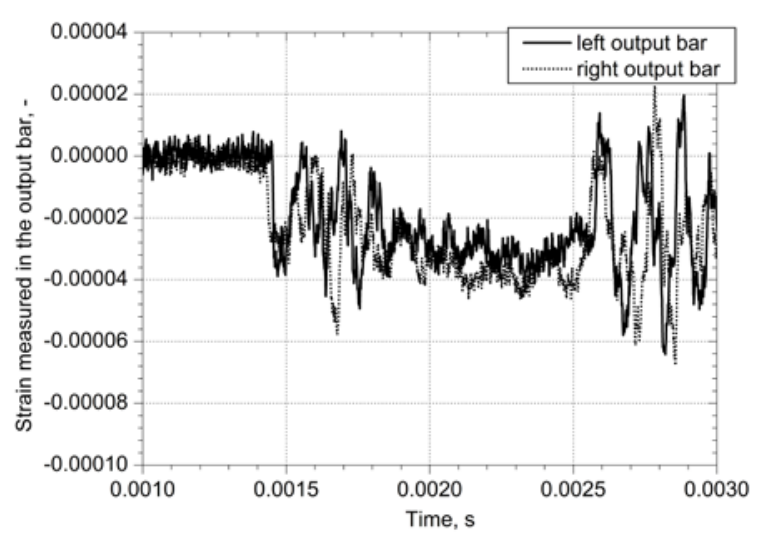

(a)

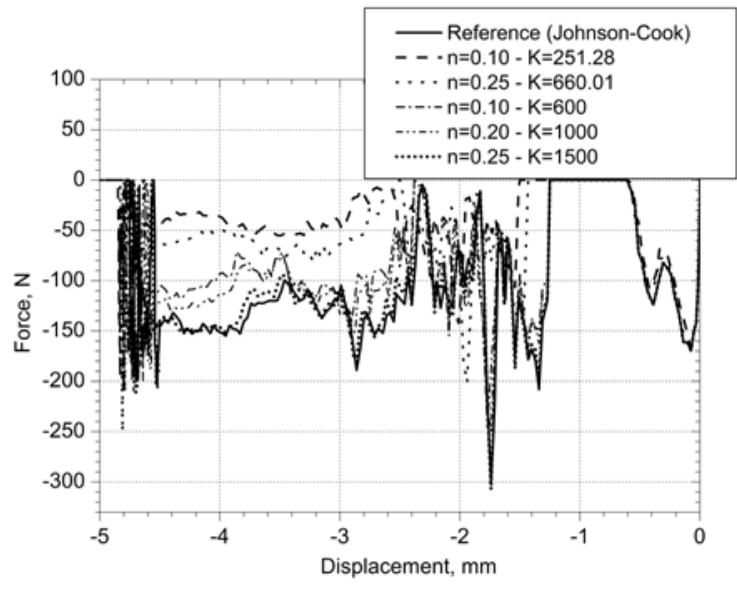

(b)

Figure 7: Experimental strain signals in both output bars of the SH3pbB setup for an industrial Sibased TRIP steel (ArcelorMittal) (a) and force applied at the input bar interface in function of the displacement for different material models (b)

\section{Summary}

In this article some aspects are highlighted of the research on the behavior of TRIP steels by making advanced use of a split Hopkinson bar setup. An integrated numerical and experimental approach is presented. The results show that TRIP steels show excellent energy-absorption properties. Yet, little difference is observed when comparing material properties in the dynamic strain rate range. The elongation of the ferritic and austenite constituents is found to be strain rate dependent and the strain induced martensitic transformation occurs gradually in the material. Advanced experimental setups provide excellent tools for validation of the material and structural properties of TRIP steels. Special sandwich specimens for SHPB experiments are developed and a Hopkinson three point bending bar setup is used to study the behavior close to the real deformation conditions of structures.

\section{References}

[1] Information on http://www.ulsab.org

[2] B. Hopkinson: Phil. Trans. A Vol. 213 (1914), p. 437

[3] V. Zackay, E. Parker, D. Fahr and R. Bush: Transactions ASM Vol. 60 (1967), p. 252

[4] P.Jacques, J. Ladriere and F. Delannay: Metall Mater Trans A Vol. 32 (2001), p. 2759

[5] H. Kolsky: Proc. Phys. Soc. Vol. 62 (1949), p. 676

[6] J. Van Slycken: Advanced Use of a Split Hopkinson Bar Setup - Application to TRIP Steels (PhD thesis Ghent University, Belgium 2008).

[7] S. Nemat-Nasser, J.B. Isaacs and J.E. Starett: Proc. R. Soc. Lond. A Vol. 435 (1991), p. 371

[8] J. Van Slycken, P. Verleysen, J. Degrieck, J. Bouquerel and B.C. De Cooman: Met. Mater. Int. Vol. 13 (2007), p. 93

[9] J. Bouquerel: Modelling and Characterisation of Multiphase TRIP Steel under Static and Dynamic Loading (PhD thesis Ghent University, Belgium 2008).

[10]G.R. Johnson and W.H. Cook, in: Proceedings of the 7th International Symposium of Ballistics, The Hague, The Netherlands (1983), p. 541

[11] J. Hollomon: AIME Transactions Vol. 162 (1945), p. 268 